\title{
EDITORIAL
}

\section{Fundamental research in nanoscience, a necessity}

How well do we understand matter and its organization at the scale of single atoms and molecules? Do we understand all driving forces and the effect of collaborative actions well enough, to actually build devices to exact specifications? Every nano-scientist today will probably answer the first question with "Not too well", and the second one with a straightforward "No". The answers will become clear, if one studies the original research contributions of a number of outstanding international scientists contained in this special issue. It is not so much our inability to predict ordering processes necessary for a fabrication of nanostructures and nanodevices, it is rather the extremely large flexibility of matter to organize itself, once different pathways to its organization become equivalent from an energy point of view. In this respect research has witnessed a gradual rapprochement over the last decade between top-down and bottom-up methods. While top-down methods become increasingly precise and their resolution limits smaller and smaller, bottom-up methods are increasingly able to lead to one particular and well defined structure at the atomic or molecular scale. This rapprochement gradually removes both, the weakness of top-down methods, which were seen as too imprecise to really be able to produce nanoscale structures, and the weakness of bottom-up methods, which were seen as too narrow to produce these structures to desired specifications. However, as the answers above make amply clear, we are not yet at the point, where both methods could be used in tandem to produce any structure or device to specifications imposed from the outset.

This is the reason, that fundamental research in this area is so important. Science, let us add, is not technology, and the frontiers of science are as far removed from industrial technology as one can think. As the authors of these articles show, it is quite common that simple and seemingly well understood systems in combination reveal, upon a more careful analysis, features and behavior which have not been encountered before. For scientists, working in this field, it makes their endeavor highly stimulating and intellectually rewarding. For the readers of this special issue, it should provide some insight how matter organizes and interacts at the nanoscale, as well as an appreciation, how difficult the fabrication of well defined nanodevices is even today.

Frontiers of Physics in China gathers together experts in the field on nanoscience for this special issue. Nine excellent papers present recent progress in the analysis, the understanding, and the fabrication of nanoscale devices. Karl-Heinz Ernst sheds lights on intermediates in molecular self-assembly; Hong-wang Zhang and coworkers describe routes to fabrication and assembly of magnetic particles; Qin Liu and coworkers study surface dy-

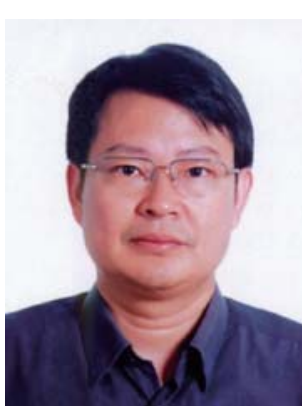

Prof. Hong-jun GAO

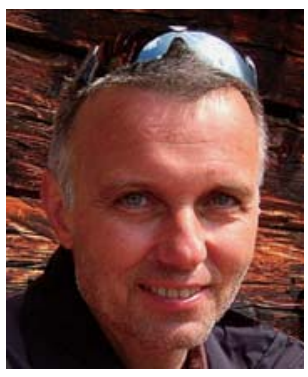

Prof. Werner A. HOFER

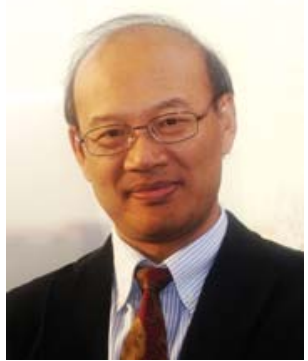

Prof. En-ge WANG namics; Haiping Lin and colleagues describe a new theoretical method to simulate tunneling currents; Shi-xuan Du and coworkers explain rotation of single molecules at surfaces; Hai-feng Ma and colleagues find new applications for a boron-nitride nanomesh; Li-feng Yin and Jian Shen tailor magnetic nanodots; Min Gao and coworkers find a method to characterize optoelectronic nanostructures in situ; finally, Ru Huang and Run-sheng Wang analyze silicon nanowire transistors in view of their application as the ultimate CMOS technology.

While more and more of nanoscience transforms into new technologies, this special issue certainly shows that its scientific basis, the understanding of events at this scale, is not in danger of drying out. Quite to the contrary, the field is expanding and becomes ever more exciting.

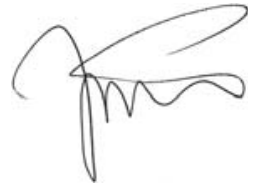

IOP, CAS, China

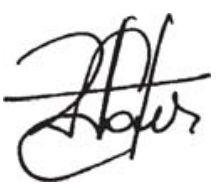

The University of Liverpool, UK

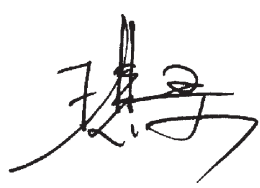

Peking University, China 